\title{
Il programma italiano di ricerche nella alta atmosfera
}

\author{
L. BRogLro \\ Riceruto il 15 Giugno 1961
}

INTRODUZIONE.

Lai Commissione per le Ricerche Spaziali, nominata dal Consiglio Nazionale delle Ricerche diciotto mesi fa, ha intrapreso un programma di stndio dell'alta atmosfera a mezzo di rizzzi sonda. Questa ricerca, (he nella attuale prima fase di laroro e sviluppo dere essere consideratia una delle attività basilari della Commissione, avera ed ha un duplice scopo: uno scopo diretto, immediato, ehe consiste nell'acquisizione di dati scientifici, in maggior copia possibile, tali da ampliare ed approfondire le attuali conoscenze sulla struttura e sulla dinamica della alta atmosfera.

Il seconclo scopo è, se cosi si può dire, proiettato nel futuro. L'organizzazione predisposta, sia in uomini che in attrezzature ed installazioni, per questa ricerca può considerarsi infatti come il nocciolo di una più ampia organizzazione the potrà operare negli anni successivi per maggiori e più impegnativi compiti.

I fondi necessari per lo sviluppo del progrumma di ricerche nell'alta atmosfera sono stati forniti alla Commissione delle Ricerehe Spaziali in base ad un accordo fra il C.N.R. e il Comitato Razzi e Missili del Ministero della Difesa, secondo il quale i due Enti si impegnarano a contribuire alle spese necessarie su base paritetica.

Il C.N.R. stipuló anche un aceorlo di natura scientifica con l'Ente spaziale statunitense N.A.S.A., sia per ottenere dalla Commissione delle Ricerche Spaziali un appoggio tecnico, sia per altre finalità di cui si parlerà più diffusamente in seguito. 
LE CARATTERISTICHE SCIENTHFICIIE E TECNICIE DEL PROGRAMMA.

Ise attuali conoscenze dell'alta atmosfera sono abbastanza scarse, in particolare nel periodo invernale, e questo sia dal punto di vista della dinamica (venti) che della "struttura" (densità, temperatura, ecc.) dell'atmosfera stessa. Inoltre è fino ad oggi mancata ogni correlazione fra le esperienze e le ricerche compiute in varie parti della terra, cosicché non si ha alcuna informazione sulla "dinamica globale " dell'atmosfera.

Per quanto riguarda le ricerche con razzi sonda l'intervallo che oggi appare più proficuo per la sperimentazione è fra le quote (intese come ordine di grandezza) di $60 \mathrm{~km} \mathrm{e} 300-400 \mathrm{~km}$.

Al di sotto dei $60 \mathrm{~km}$ il campo è da considerarsi acquisito ai normali mezzi di indagine meteorologica (palloni sonda, razzi meteorologici, ecc.). Sopra i $300 \mathrm{~km}$ invece sono probabilmente i satelliti gli strumenti tecnici più adatti allo scopo: resta comunque un ampio intervallo di quote in cui, allo stadio attuale della tecnica, sembrano poter operare convenientemente i soli razzi sonda.

Quale tecnica da impiegare nella ricerca fu scelta quella della nube di sodio e litio. Essa presenta il vantaggio di coprire con un solo lancio un intervallo notevolmente ampio di quote e di fornire informazioni e dati contemporaneamente sulla struttura e sulla dinamica dell'atmosfera. Come è noto, in una esperienza del genere il carico utile del razzo sonda è costituito da una miscela di una sostanza esotermica - capace cioè di gencrare calore per autocombustione - (termite, ad es.) e sodio con delle particelle addizionali di litio distribuite in seno alla massa maggiore.

La combustione di una carica iniziale di termite produce quella della miscela termite-sodio. Il sodio, vaporizzato, fuoriesce, attraverso le apposite aperture praticate nel contenitore, in modo continuo, mentre ad intervalli, generalmente irregolari, viene espulso "a sbuffi " il litio, parimenti vaporizzato. In tal modo il sodio ha nell'aria circostante una diffusione quasi-cilindrica, mentre il litio ha una diffusione quasi-sferica.

\section{LE A'TTREZZATURE AL SUOLO.}

Quale base di lancio per i razzi sonda fu scelto il Poligono del Salto di Quirra (Perdasdefogu-Sardegna) (Fig. 1), ma per il programma della Commissione delle Ricerche Spaziali si provvide a realizzare delle installazioni nuove e quasi del tutto indipendenti dalla già esistente base militare.

Nello stesso tempo furono realizzate le stazioni di osservazione che dovevano fotografare la nube di sodio. Il criterio di scelta delle località 


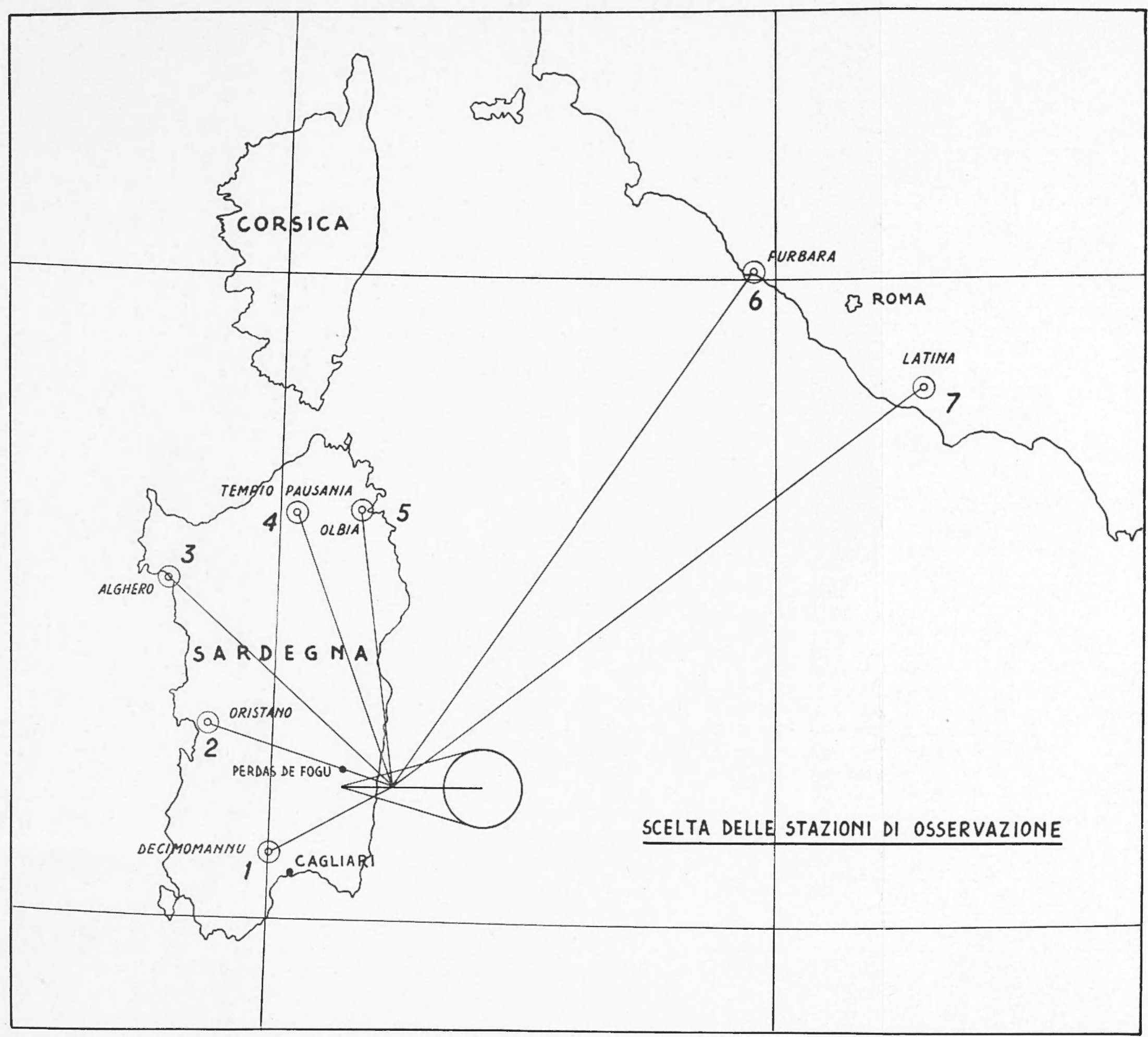

Fig. I 
ove installare le apparecchiature fotografiche di ripresa fu essenzialmente quello di ottenere sui fotogrammi immagini della nube di considerevoli dimensioni senza peraltro che queste divenissero tanto grandi da non rientrare nei fotogrammi stessi. Furono anche tenuti presenti altri fattori, quali quelli puramente logistici.

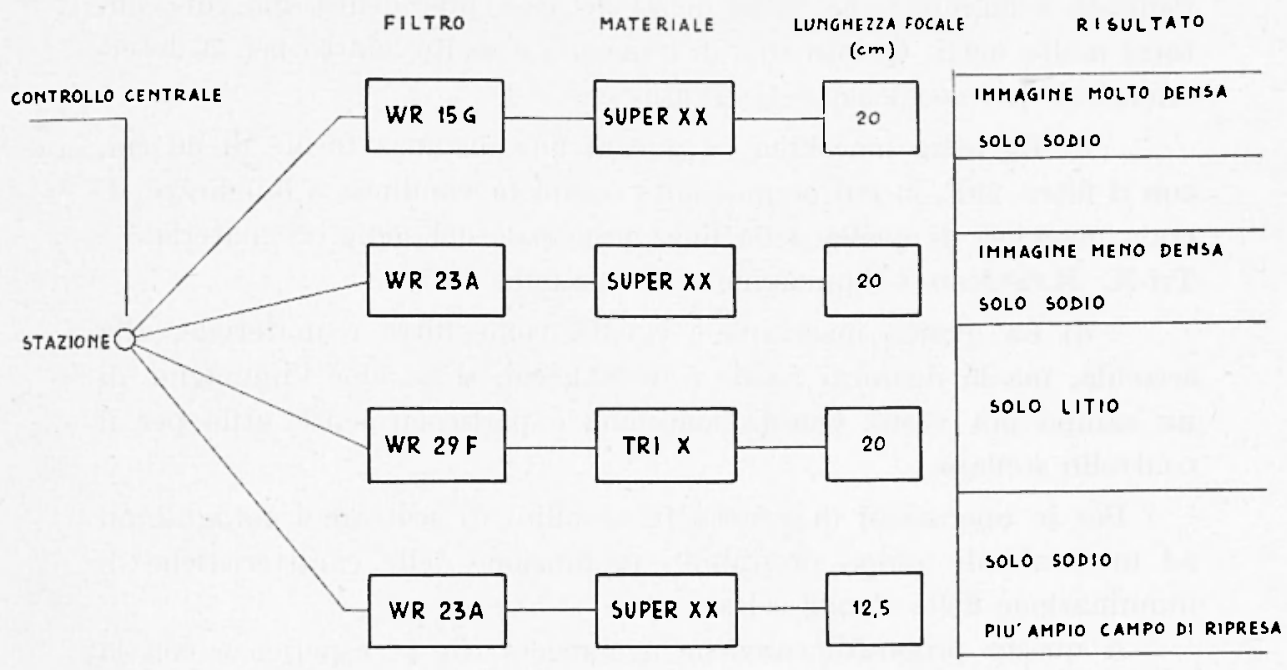

\section{FILTRI}

WR $15 G$ - PERMEABILITA' COMPLETA DA $\lambda=3600 \mathrm{~A}$

WR $23 A$ - PERMEABILITA COMPLETA POCO PRIMA DI $\lambda_{\text {Ma }}$

WR $29 \mathrm{~F}$ - PERMEABILITA COMPLETA PER $\lambda>\lambda_{\mathrm{Ha}}$

\section{MATERIALI}

SUPER $X X$ - NON VALIDO PER IL LITIO

TRI $X$ - VALIDO PER IL LITIO

Fig. 3

Quanto detto sopra vale per le stazioni installate in Sardegna; per quelle continentali invece lo scopo è essenzialmente quello di fotografare la nube contro uno sfondo celeste di diversa illuminazione rispetto a quello delle stazioni in Sardegna. L'elevato numero di stazioni, sette in tutto, è giustificato dalla necessità di ottenere una elevata "reliability " globale. In Fig. 1 è indicata la disposizione delle stazioni.

Ciascuna stazione è dotata di quattro macchine fotografiche montate su di un supporto comune, come indicato in Fig. 2. Le caratteristiche principali delle macchine sono le seguenti (Fig. 3):

a) La prima macchina ha una distanza focale di $20 \mathrm{~cm}$ con $\mathrm{mn}$ filtro 15 G a permeabilità totale sopra 3600 A; il materiale è Super XX, 
valido per il sodio, ma non per il litio. Il risultato è un'immagine molto densa della sola nube di sodio.

b) Ia seconda macchina ha una distanza focale di $20 \mathrm{~cm}$, con il filtro $23 \mathrm{~A}$ ehe ha permeabilità completa proprio immediatamente prima della linea principale del sodio. Il materiale è ancora Super XX. Il risultato è un'immagine meno densa del caso precedente, ma con contorni molto netti. Questo tipo di immagini è molto adatto per la determinazione del coefficiente di diffusione.

c) Ia terza macchina ha ancora una distanza focale di $20 \mathrm{~cm}$, con il filtro $29 \mathrm{~F}$, la cui permeabilità completa comincia a lunghezze di onda maggiori di quella della linea principale del sodio. Il materiale ̀̀ Tri-X. Il risultato è l'immagine della sola nube di litio.

d) Isa quarta macchina è eguale, come filtro e materiale, alla seconda, ma la distanza focale è di $12,5 \mathrm{~cm}$, si ha cioè l'immagine di un campo più vasto. Questa macehina è particolarmente utile per il controllo stellare.

Per le operazioni di ripresa fu stabilito di seattare $\mathrm{i}$ fotogrammi ad intervalli di tempo prestabiliti in funzione delle caratteristiche di illuminazione dello sfondo celeste.

A questo proposito conviene agriungere che le esperienze con la nube di sodio possono essere compiute solo allalba o al tramonto, in quanto, mentre la nube — per poter essere ripresa — deve essere illuminata dai raggi del sole, lo machine fotografiche al suolo non devono essere impressionate direttamente dal sole, o anche indirettamente da una illuminazione dello sfondo troppo intensa, perché l'immagine della nube risulterebbe "ammegata ».

Questo vincolo implica che le esperienze con la nube di sodio possono compiersi solo in un ristretto intervallo di tempo (o all'alba, o al tramonto), da cui deriva un notevole complicazione nelle esperienze stesse in quanto è necessario evitare qualunque inceppamento, non brevissimo, nelle operazioni di "count-down", pena il rinvio del lancio di ameno dodici ore.

\section{IL PRIMO LANCIO.}

Il primo lancio italiano di un razzo sonda ebbe luogo, con pieno successo il 12 Gennaio di quest'anno alle 17,54 (tramonto). Questo esperimento fu inteso fondamentalmente come prova generale dell'organizzazione predisposta per il programma. 
A questo scopo fu scelto, come vettore, la combinazione Nike-Cajun, di prestazioni non eccezionali - la massima quota raggiungibile è dell'ordine di $150 \mathrm{~km}$ - ma di elevata " reliability ».

Un lavoro preliminare, condotto all'Istituto di Costruzioni Aeronautiche dell'Università di Roma, consistette nella valutazione della traiettoria del razzo. La traiettoria reale, desunta dalle riprese a terra (Fig. 4), ha ben confermato le previsioni teoriche.

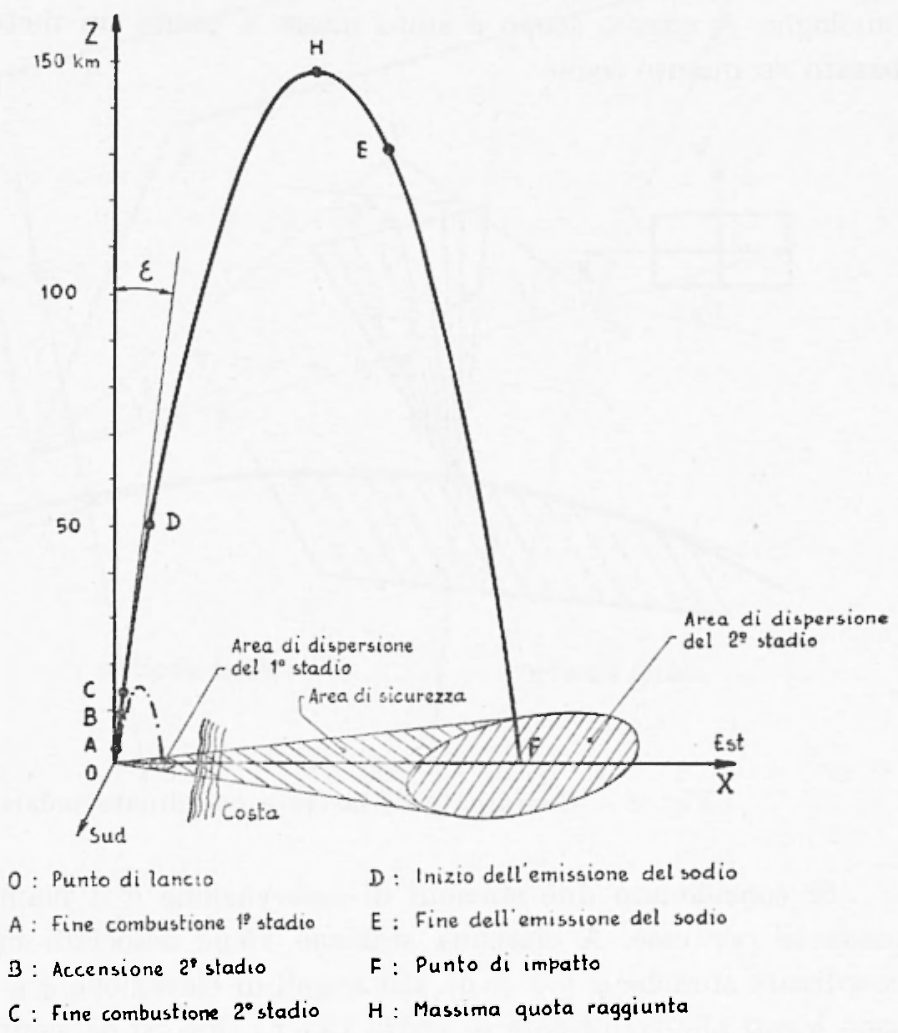

Fig. 4

Si è detto che lo scopo principale di questo lancio era di natura pratica in quanto si voleva valutare, in fase operativa, l'organizzazione predisposta. Questo non toglie nulla, però, all'interesse scientifico dell'esperienza, tenendo in particolar modo presente che essa è stata realizzata in un periodo dell'anno in cui ben poco si sa dello earatteristiche dell'alta atmosfera. La Fig. 5 (ripresa dalla stazione di Oristano) mostra la nube di sodio. La Fig. 6 è un particolare della precedente che mette in 
evidenza l'importantissimo fenomeno della turbolenza. La Fig. 7 infine mostra un confronto fra le nubi di litio (a destra) e di sodio.

\section{LA RESTITUZIONE DEI DA'TI.}

La difficoltà principale nella restituzione dei dati ricavati da una esperienza con la nube di sodio consiste nella individuazione dei punti omologhi. A questo scopo è stato messo a punto un metodo analitico basato su quanto segue.

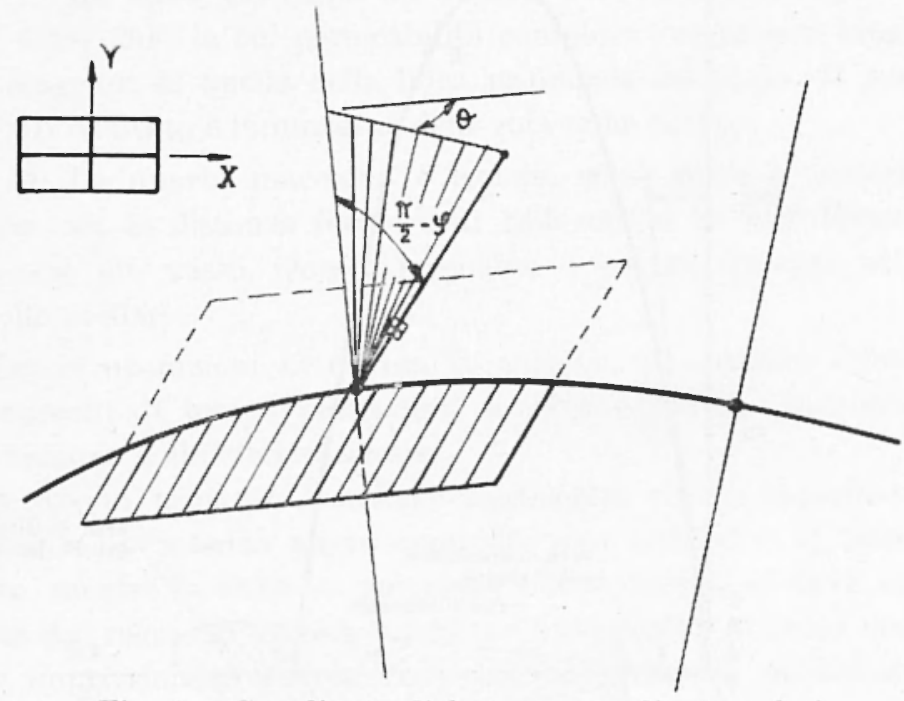

Fig. 8 - Coordinate di lastra e coordinate polari

Si considerano due stazioni di osservazione e il piano verticale a passante per esse. A ciascuna stazione viene associato un sistema di coordinate sferiche $\varphi$ e $\theta$ (e $\varrho$ ). Gli angoli di elevazione $\varphi$ e di azimuth $\theta$ sono legati alle coordinate di lastra (X, Y) (Fig. 8) da semplici relazioni trigonometrich. Queste relazioni permettono dunque di individuare ogni punto della nube vera in funzione di $\varphi, \theta$ (e della distanza $\varrho$, per il momento incognita).

D'altra parte un punto della nube può essere individuato dagli angoli $\xi$ e $\zeta$ (Fig. 9), che sono ricavabili da $\varphi$ e $\theta$ in modo assai semplice. E possibile allora ricarare per ogni stazione e per ogni immagine le curve $\xi=\xi(\zeta)$ (Fig. 10). Ora, per definizione, ogni punto della nube ha lo stesso sia che sia visto da una stazione che dallaltra ed è quindi immediato risalire ai punti omologhi. 
Una volta determinati i punti omologhi, si può risalire in modo rapido ed agevole alla posizione spaziale vera di tutti i punti della nube ad ogni istante di ripresa.

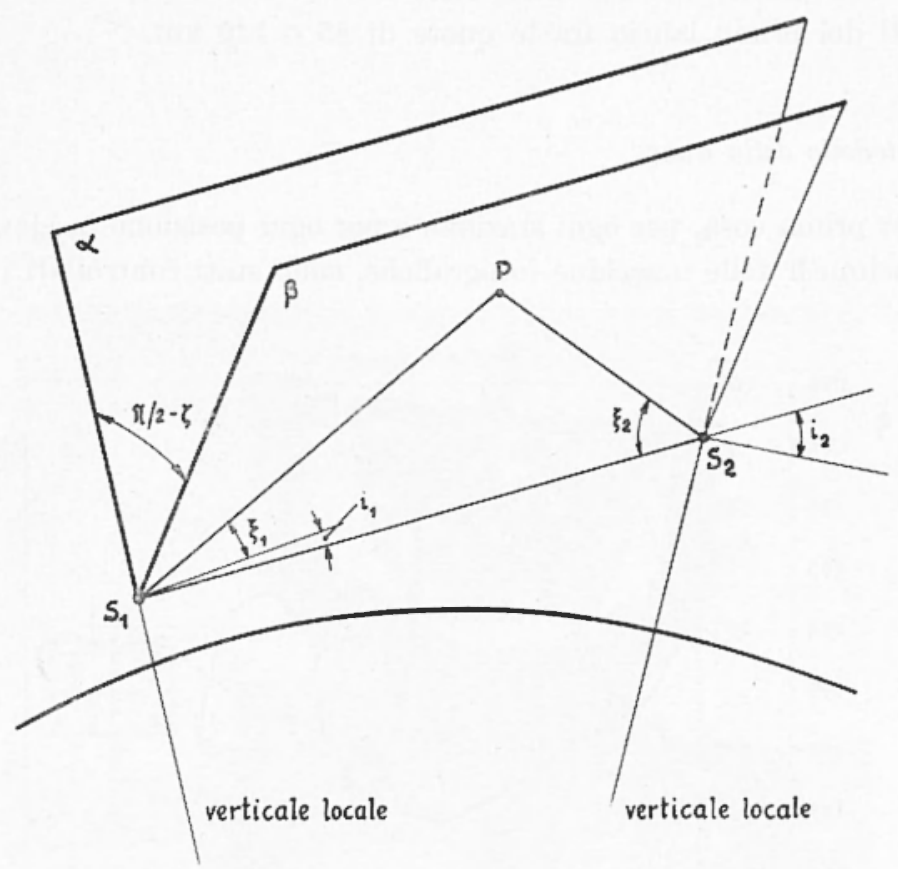

Fig. 9

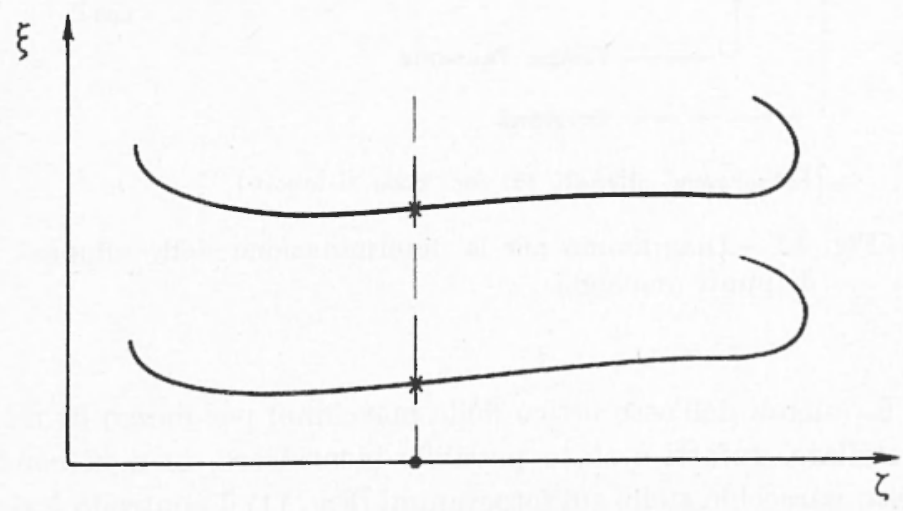

Fig. 10 - Determinazione dei punti omologhi 
I RISULTATI DEL PRIMO LANCIO.

In base al metodo sopra descritto è stata effettuata la restituzione dei dati del primo lancio fra le quote di 85 e $140 \mathrm{~km}$.

a) Il modello della nube.

Per prima cosa, per ogni stazione e per ogni posizione in elevazione ed in azimuth delle macchine fotografiche, sono stati controllati i valori

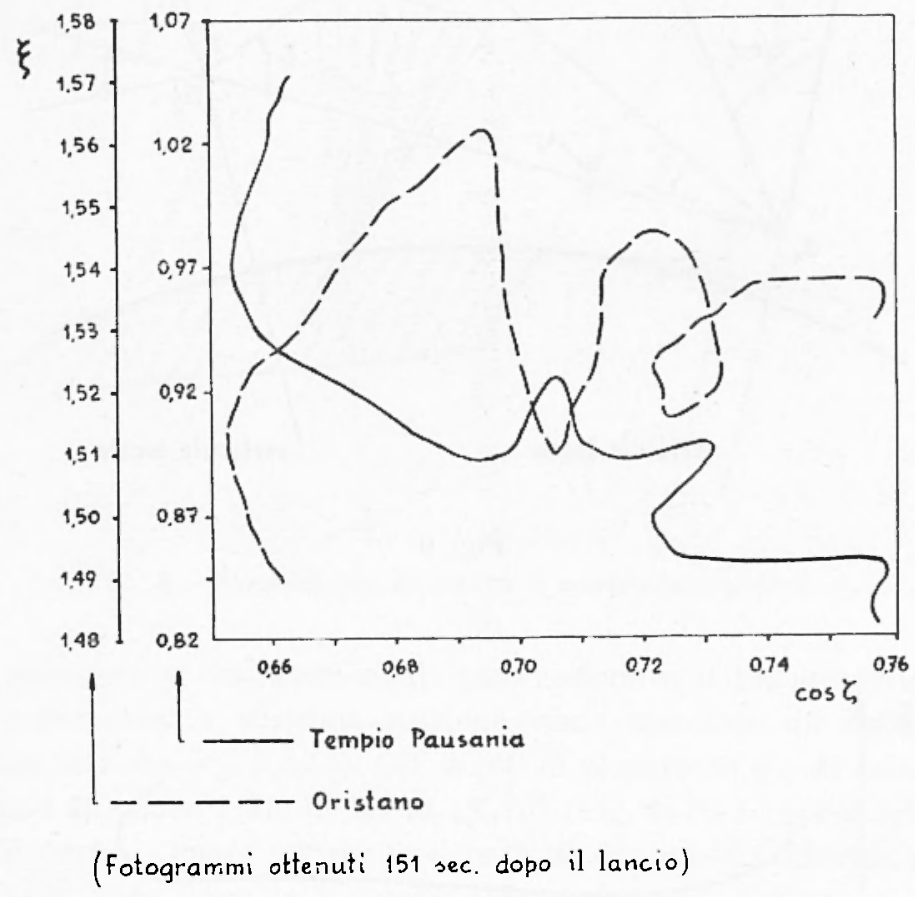

Fig. 12 - Diagramma per la determinazione delle coppie di punti omologhi.

di $\varphi_{0}$ e $\theta_{0}$ (angoli dell'asse ottico delle macchine) per mezzo di un riferimento stellare. Infatti è stato possibile identificare, in ogni condizione di ripresa, parecchie stelle sui fotogrammi (Fig. 11) il controllo è risultato del tutto soddisfacente. 
L. BROGLIO

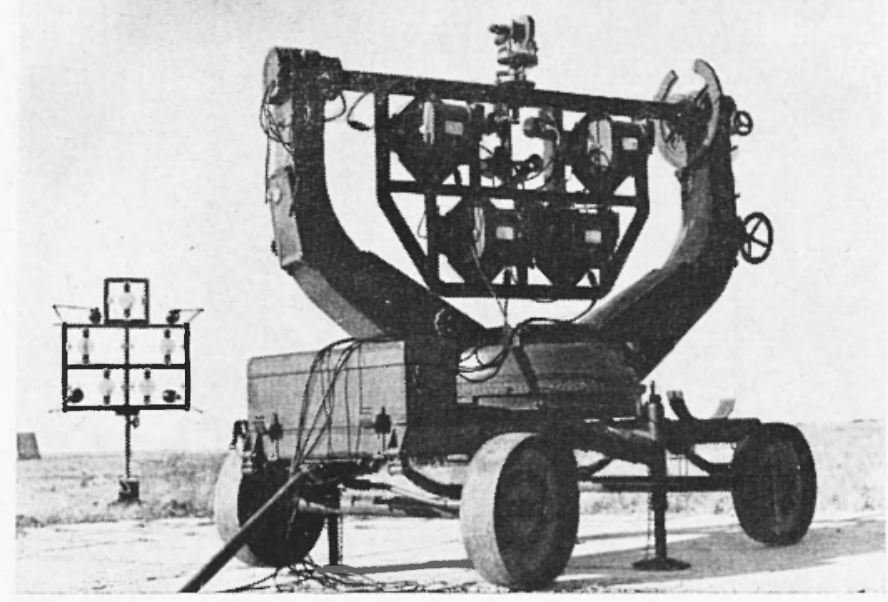

Fig. 2 
L. BROGLIO

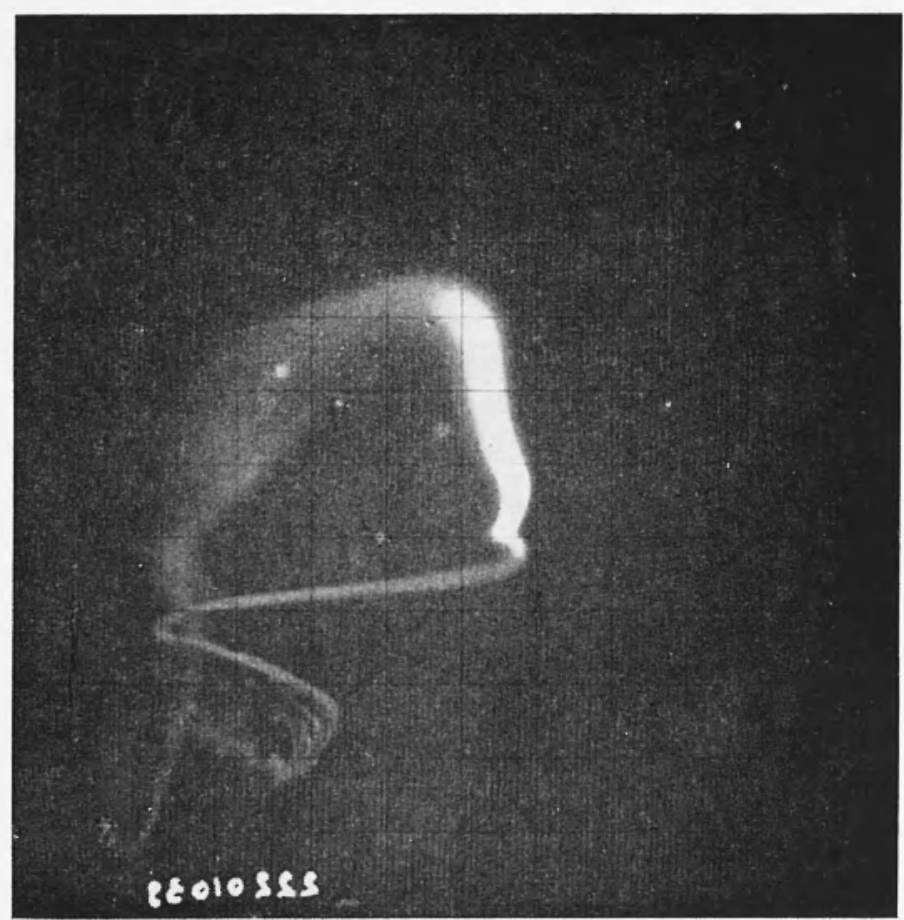

Fig. 5

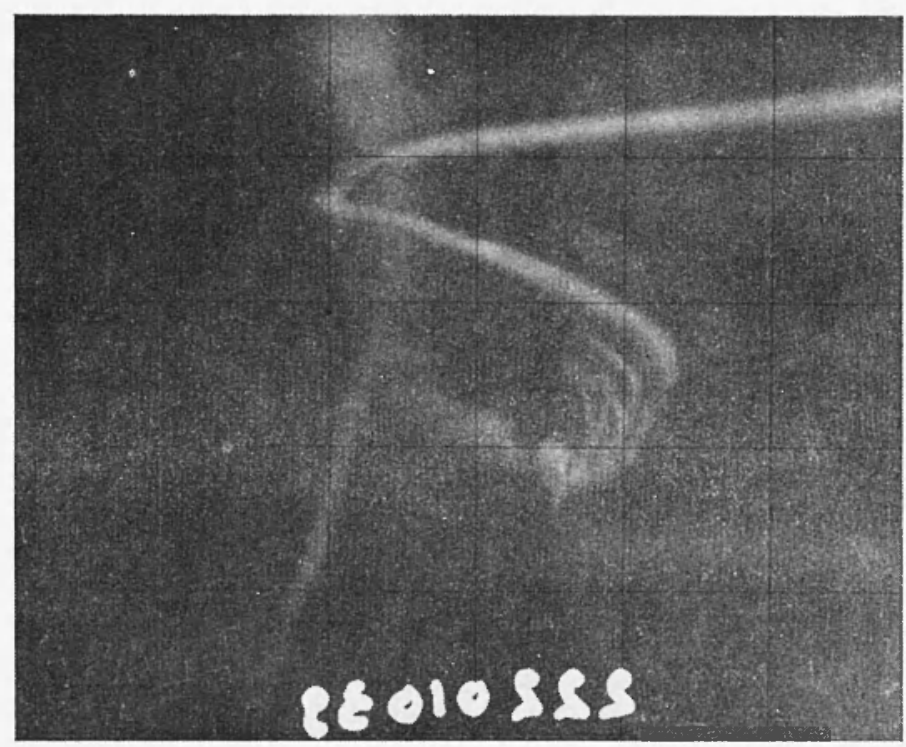

Fig. 6 


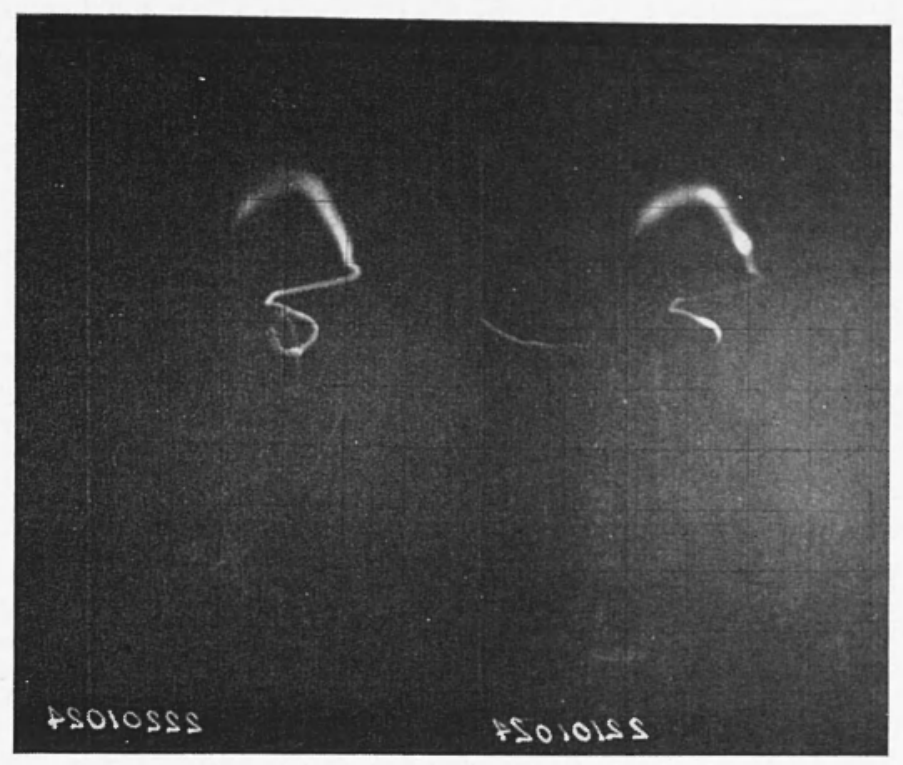

Fig. 7

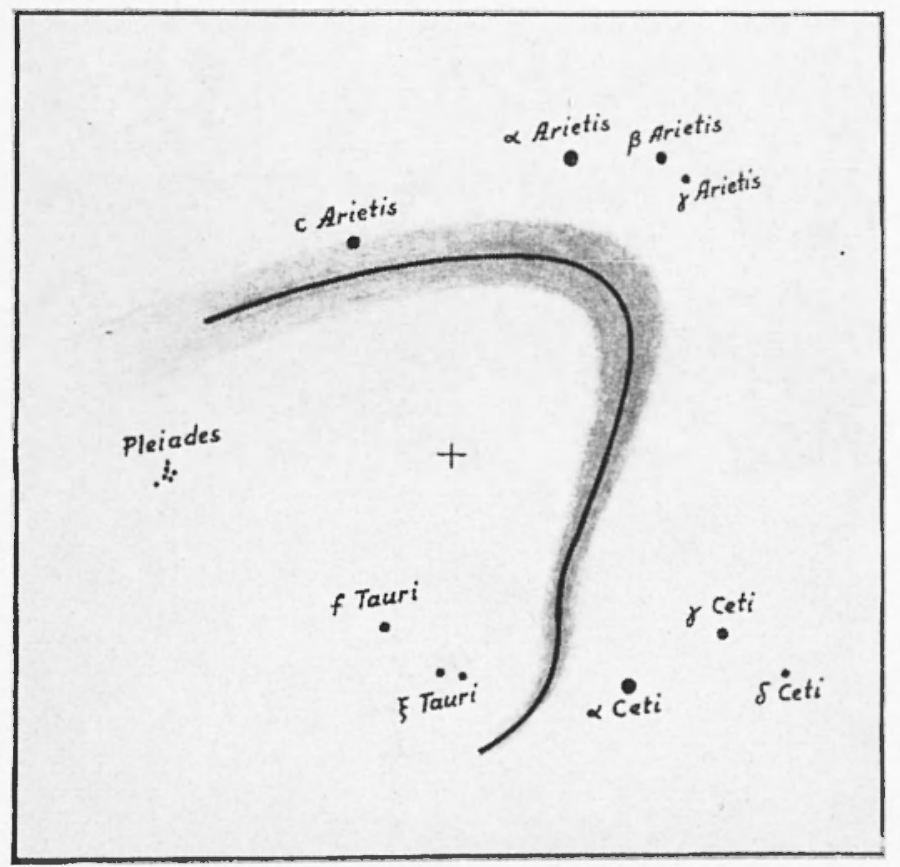

Fig. 11 - Stazione di Oristano - Fotogramma 71 - Nube e stello (Macchina: lunghezza focale $20 \mathrm{~cm}$; filtro WR $15 \mathrm{G}$ ) 
L. BROGLIO

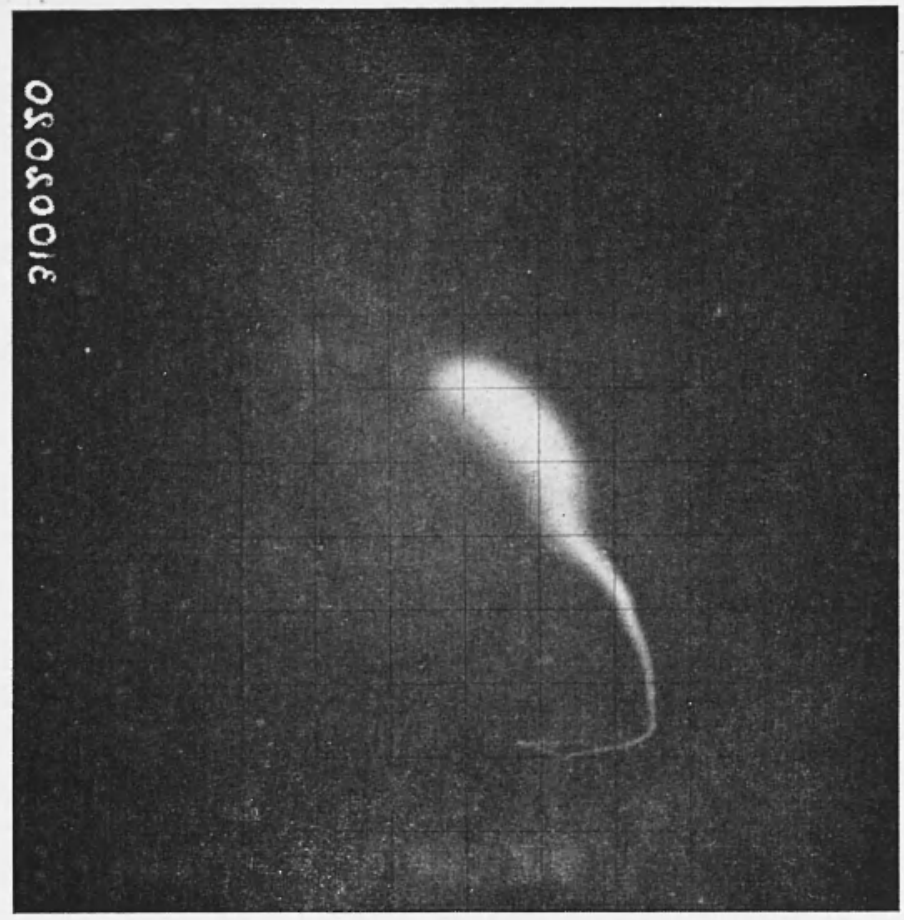

Fig. 20

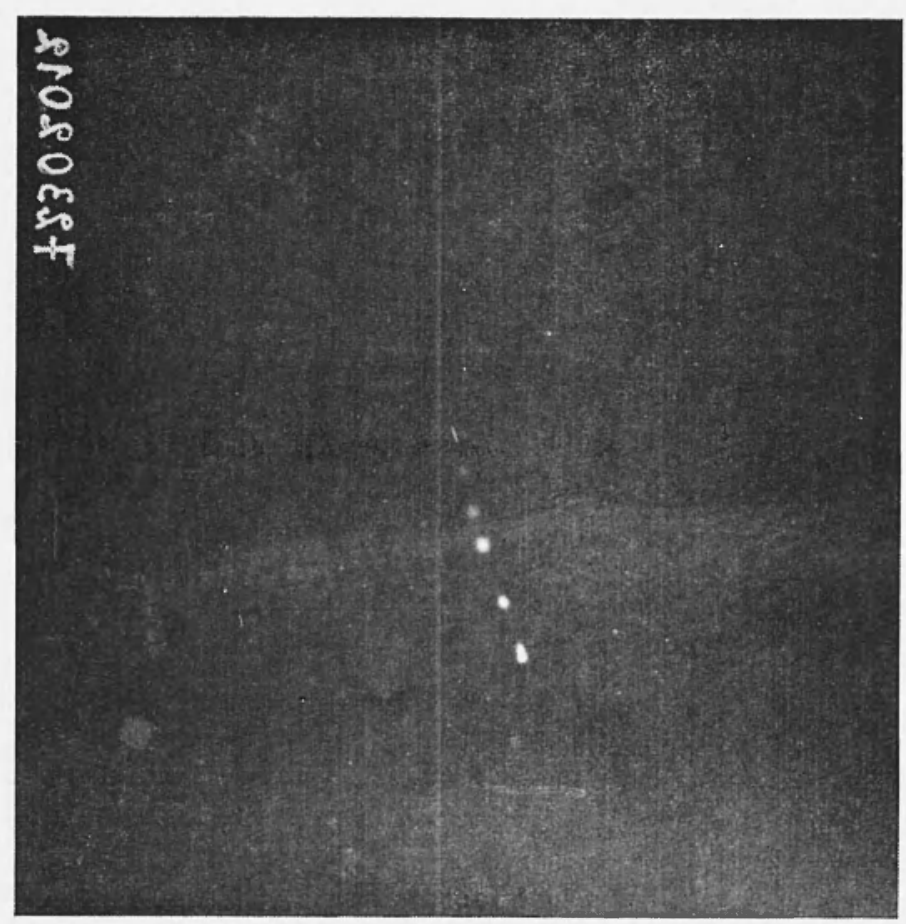

Fig. 21 
Successivamente per ogni gruppo di due stazioni furono scelti i fotogrammi corrispondenti (cioè quelli ottenuti nello stesso istante) determinando i punti omologhi. La Fig. 12 mostra, a titolo di esempio, $\xi$ in funzione di $\zeta$ per due fotogrammi ottenuti nelle stazioni di Oristano e Tempio Pausania: le curve si riferiscono all'asse centrale della nube.

b) Intensità e divezione dei venti.

Determinati i punti omologhi per ogni coppia di fotogrammi, e determinata quindi la posizione spaziale vera della nube all'istante di ripresa dei fotogrammi stessi, si è costruita la proiezione orizzontale

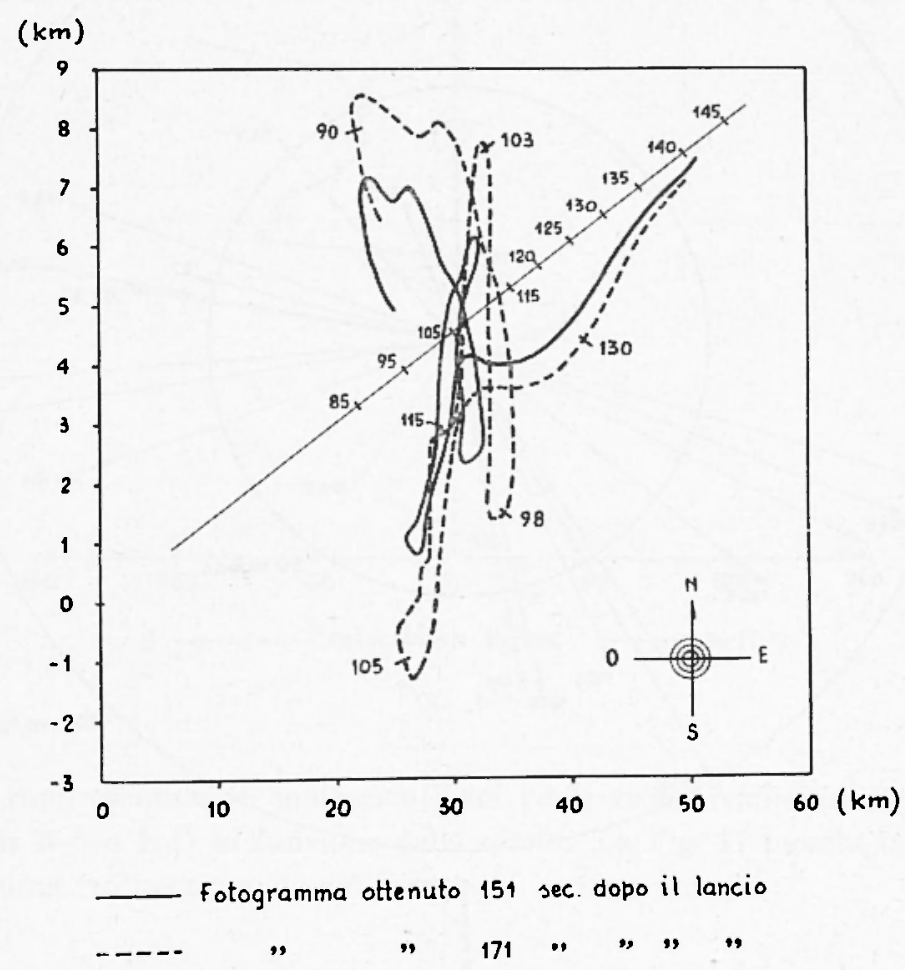

Fig. 13 - Proiezione orizzontale della nube

quotata della nube nei vari istanti: la Fig. 13 mostra, per ragioni di chiarezza di disegno, solo due curve. Da questo diagramma, in base alle ipotesi consuete sulla dinamica alle alte quote si è risaliti alla direzione e all'intensità dei venti orizzontali. 


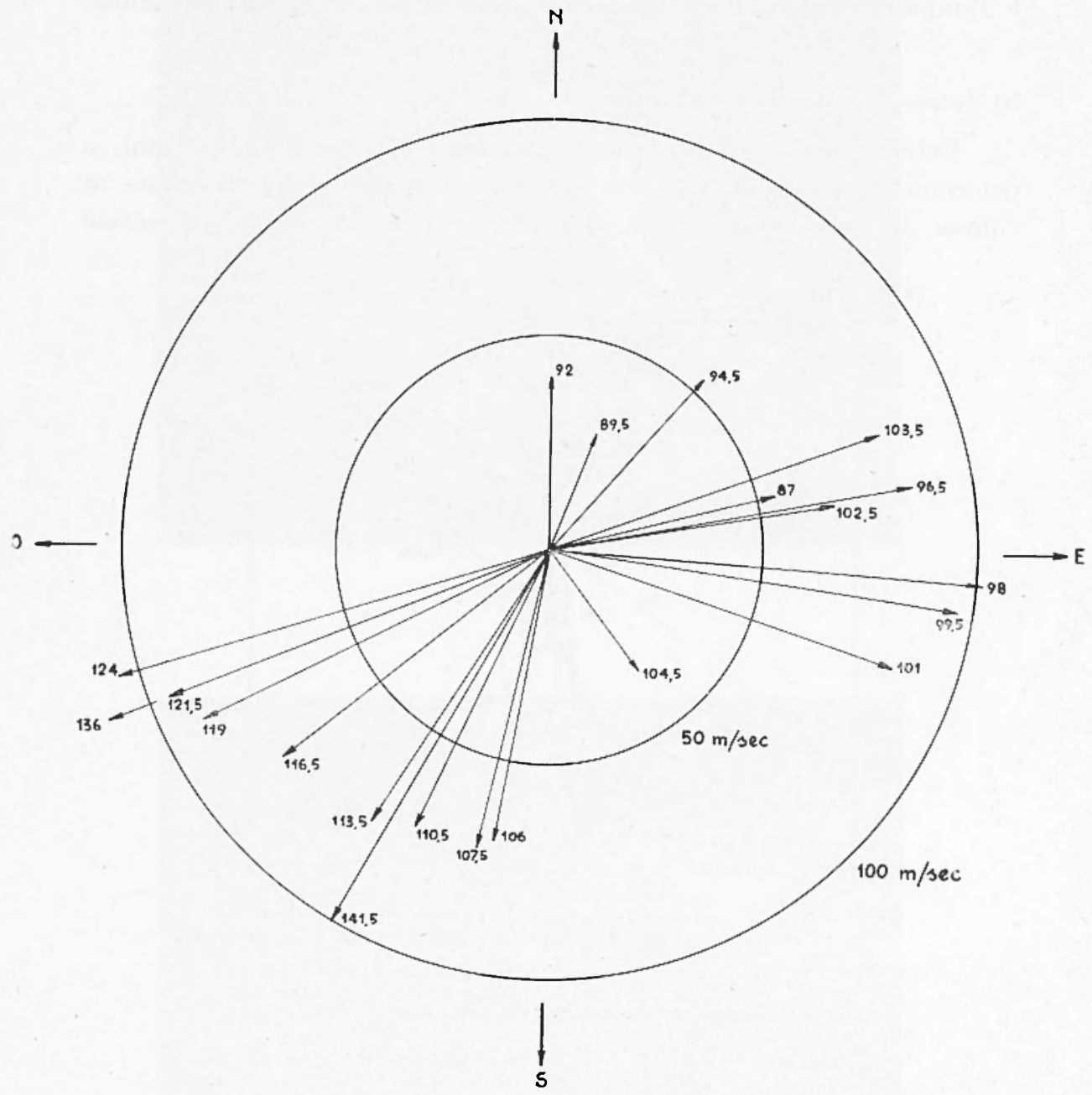

Fig. 14 - Diagramma polare della intensità $\theta$ direzione dei venti. Velocità in $\mathrm{m} / \mathrm{sec}$; quote in $\mathrm{km}$. 
Le Figg. 14, 15, 16, 17 raccolgono i risultati. Lia Fig. 14 mostra il diagramma polare dei venti fra 85 e $140 \mathrm{~km}$. Nelle Figg. 15 e 16 sono

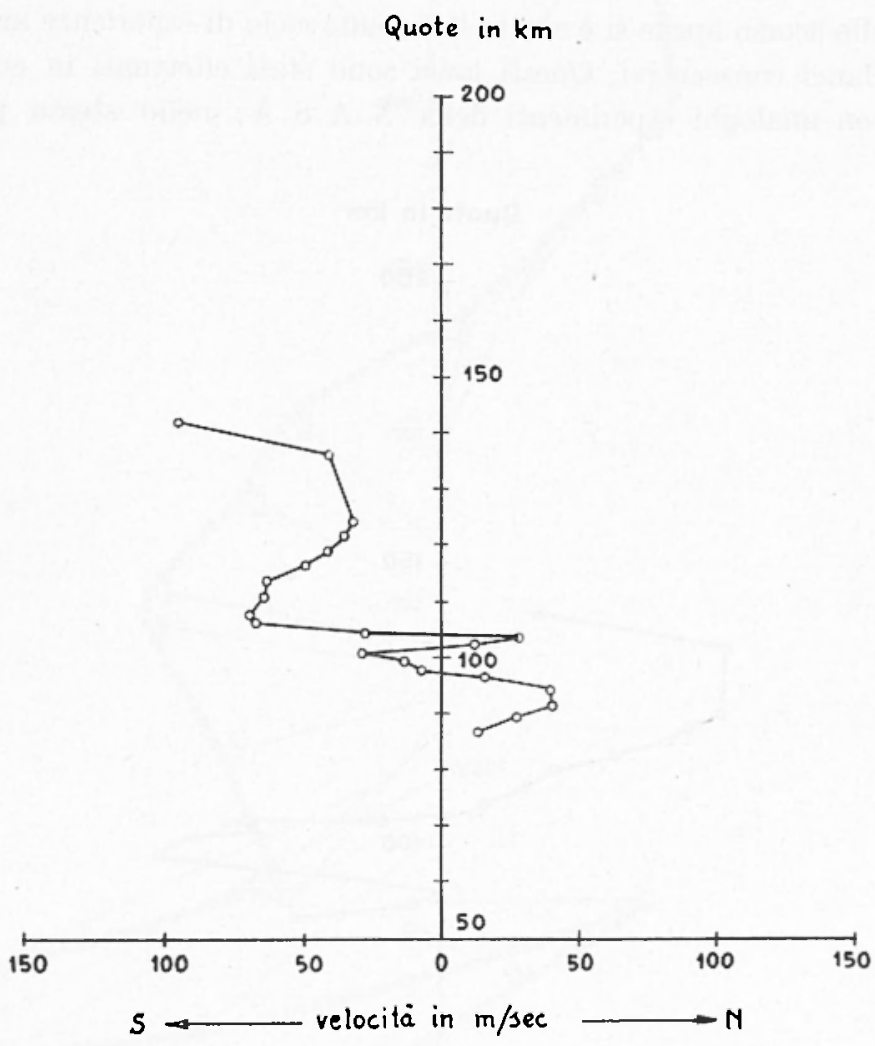

Fig. $1 \tilde{5}$

invece rappresentate le componenti dei venti rispettivamente sui piani verticali N-S e E-O in funzione della quoti. La Fig. 17 mostra infine il diagriumma tridimensionale dei venti.

c) Coefficiente di diffusione e densitù.

Il coefficiente di diffusione è stato ricuvato leggendo, mediante un microdensitometro, sull'immagine delli nube le densità fotografiche. I risultati sono riportati in Fig. 18. Dal coefficiente di diffusione si è poi risaliti alla densità (Fig. 19) in base alle formule usuali della teoria cinetica dei gas. 


\section{LA SECONDA SERIE DI LANCI.}

Nello scorso aprile si è svolto il secondo ciclo di esperienze articolate su tre lanci consecutivi. Questi lanci sono stati effettuati in coordinazione con analoghi esperimenti della N.A.S.A. nello stesso periodo.

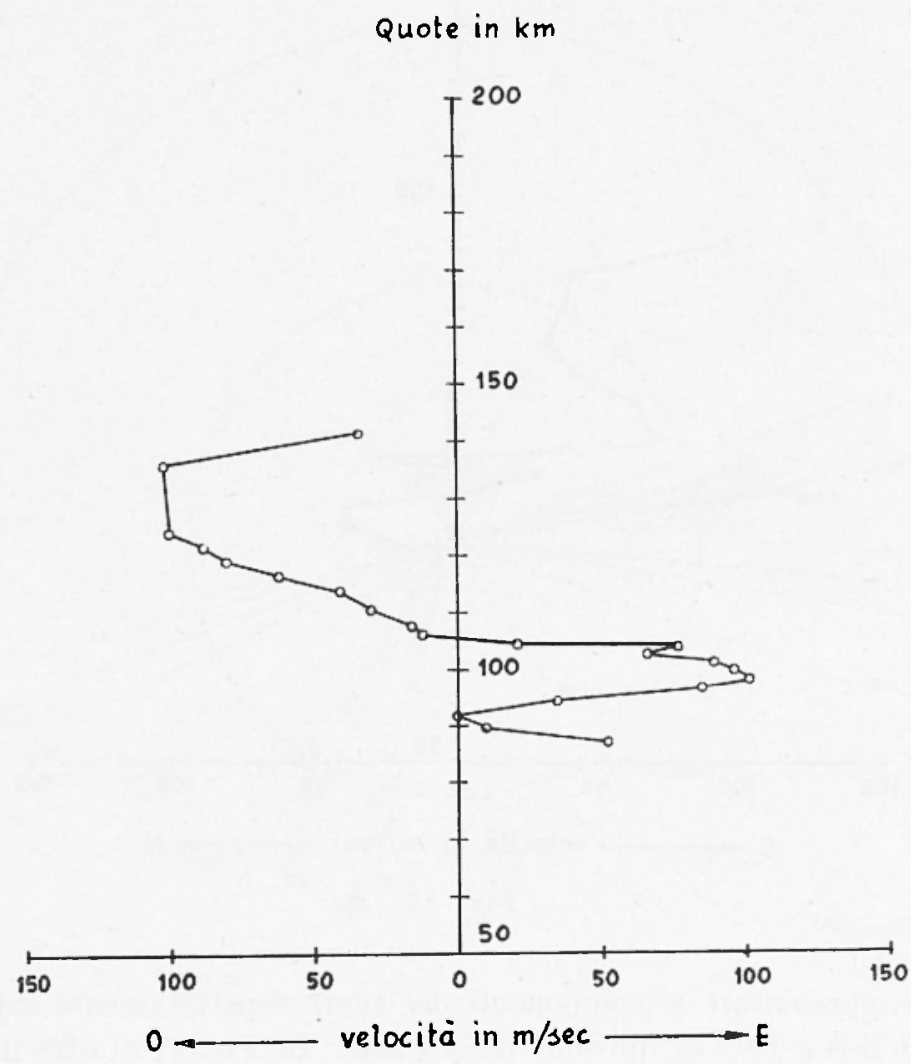

Fig. 16

Le esperienze sono state condotte tenendo presente due obiettivi principali. Il primo consisteva nello studio delle fluttuazioni di « corto periodo" sopra la zona di lancio. A questo scopo i tre lanci sono stati effettuati consecutivamente nello spazio di ventiquattro ore. E interessante notare che si è trattato del primo esperimento di questo genere con razzi sonda. 


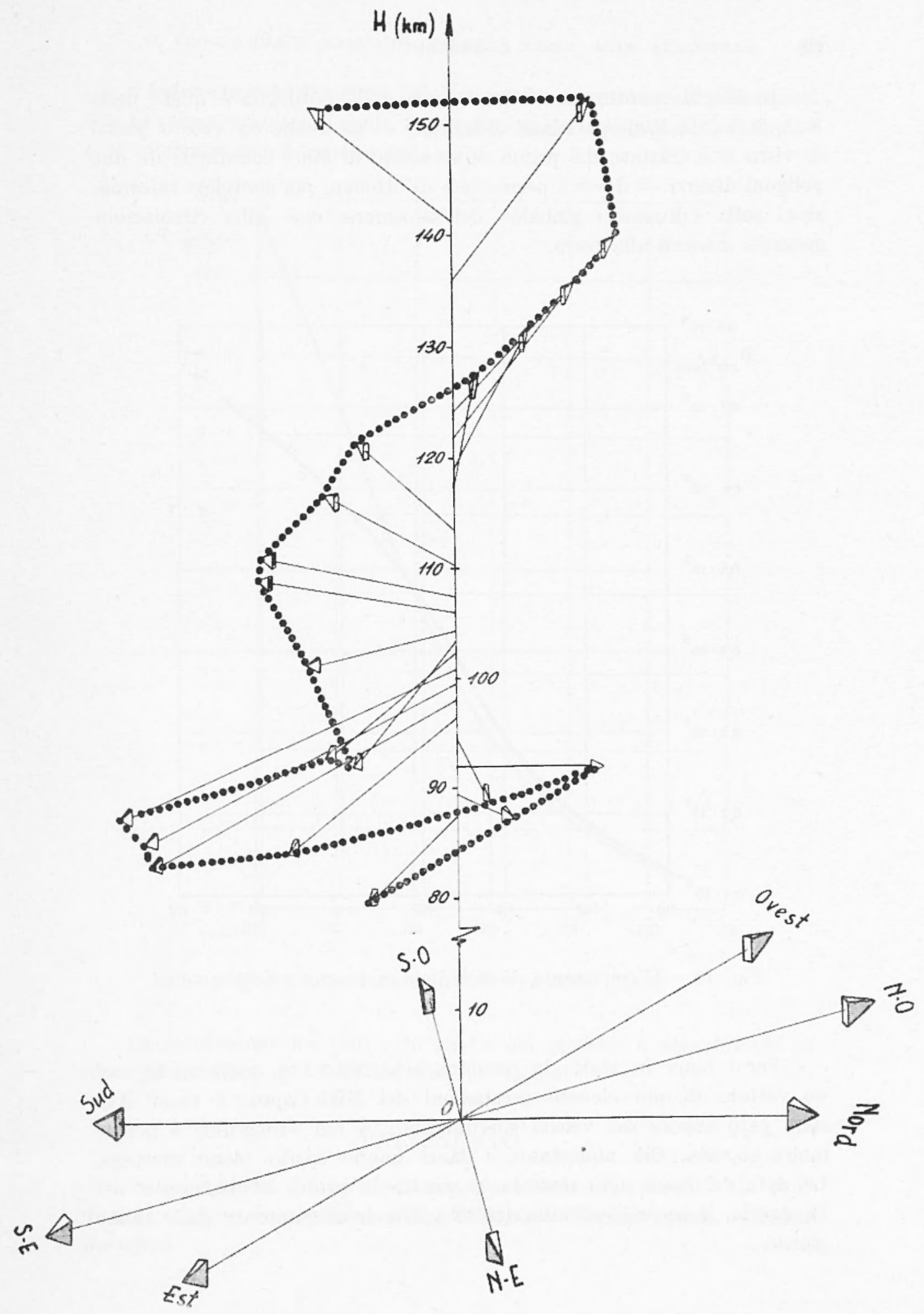

Fig. 17 - Diagramma della velocità e direzione dei venti. 
Inoltre il coordinamento fra i lanci dalla Sardegna e quelli della N.A.S.A. da Wallops Island (Virginia) - ed anche da questo punto di vista si è trattato del primo esperimento di lanci coordinati da due poligoni diversi - doveva permettere di ottenere più complete informazioni sulla "dinamica globale" dell'atmosferu, cioè sulla circolazione, generale intorno alla terra.

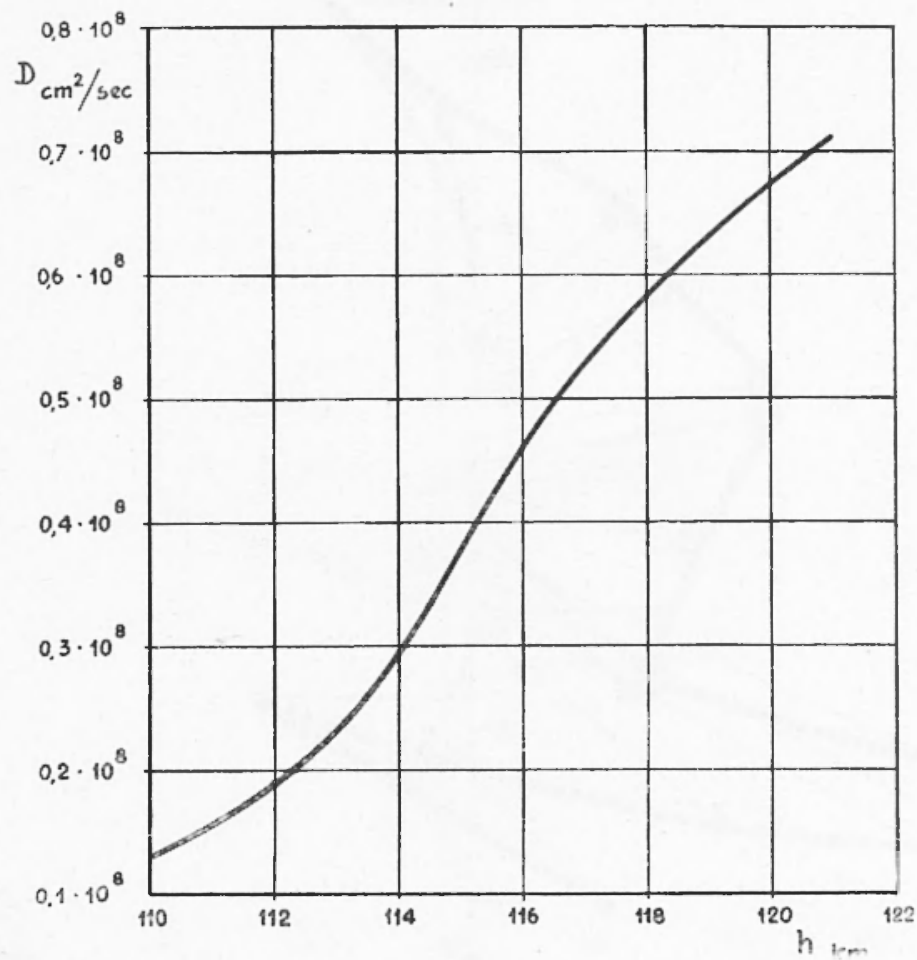

Fig. 18 - Il coefliciente di diffusione in frmzione clella quota.

Per i lanci fu scelta la combinazione Nike-Asp, realizzando così un vettore di più elevate prestazioni del Nike-Cajun. I razzi Asp sono però ancora dei veicoli sperimentali, la cui "reliability" non è molto elevata. Ciò nonostante i lanci lamno avuto pieno successo. Le date dei lanei sono state le seguenti: il primo al tramonto del 19 Aprile, il secondo all'alba del 20 e il terzo al tramonto dello stesso giorno. 
I lanci e le nubi di sodio e litio generate sono stati osservati e ripresi con lo stesso equipaggiamento fotografico usato in Gennaio. Le Figg. 20 e 21 mostrano due fotografie della nube.

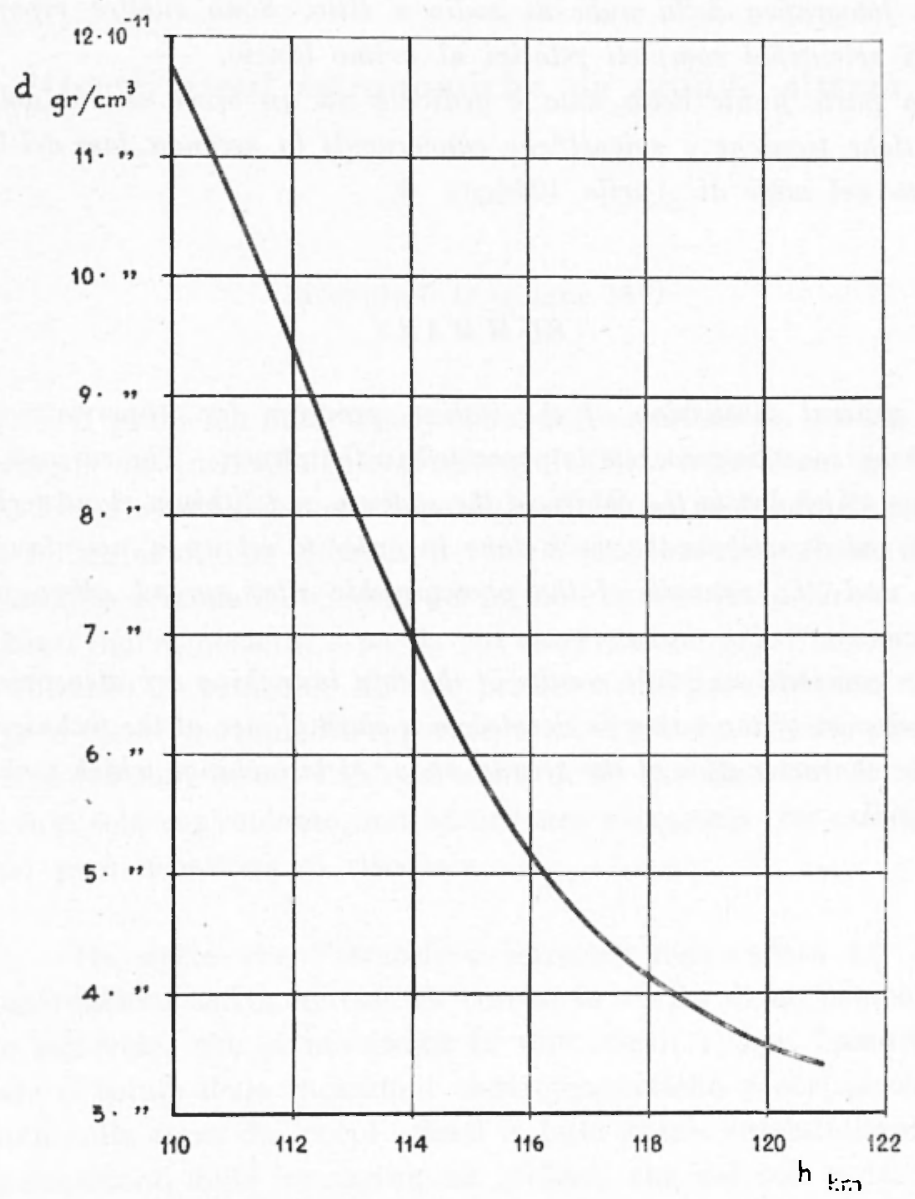

Fig. 19 - La densità in funzione della quota.

Lab restituzione dei dati e lo studio dei risultati è attualmente in corso presso l'T.C.A. dell'Università di Roma.

\section{RIASSUNTO}

In questa nota l'Autore presenta una discussione generale del programma italiano conseguito mediante l'impiego di razzi sonda nell'alta atmosfera. 
In particolare sono esposte da un lato le caratteristiche tecniche delle esperienze finora realizzate e dall'altro $i$ lavori compiuti per la realizzazione di una nuova base di lancio e della rete di stazioni per la ripresa fotografica della nube di sodio e litio. Sono inoltre riportati $i$ risultati scientifici completi relativi al primo lancio.

La parte finale della nota è dedicata ad un breve esame delle caratteristiche tecniche e scientifiche concernenti la seconda fase del lancio, avvenuto nel mese di Aprile 1961.

\section{$S U M M A R Y$}

A general discussion of the italian program for upper atmosphere research by sounding rockets is presented in the paper. The various considerations which led to the choice of the sodium and lithium cloud technique are outlined as well as the work done in order to set up a new launching facility and the network of the photographic sites spread all-round the range zone.

The complete scientific results of the first launching are also presented. The final part of the paper is devoted to a short glance at the technical and scientific characteristics of the second phase of launchings which took place last April. 